\title{
Negative Pressure Wound Therapy with Instillation and Dwell Time in the Surgical Management of Severe Hidradenitis Suppurativa
}

\author{
Shealinna $\mathrm{Ge}^{1}$, Hakan Orbay ${ }^{2}$, Ronald P. Silverman ${ }^{3}$, Yvonne M. Rasko ${ }^{1}$ \\ 1. Plastic and Reconstructive Surgery, University of Maryland School of Medicine, Baltimore, USA 2. \\ Surgery, University of Maryland School of Medicine, Baltimore, USA 3. Plastic and Reconstructive \\ Surgery, University of Maryland School of Medicine, Baltimore, USA
}

$\square$ Corresponding author: Shealinna Ge, shealinnage@gmail.com

Disclosures can be found in Additional Information at the end of the article

\section{Abstract}

Background: Hidradenitis suppurativa (HS) is a physically debilitating disease that greatly impairs the quality of life of affected individuals. Advanced disease is often difficult to treat with topical and systemic therapies. Surgical resection of diseased skin has become paramount in HS management but proposes challenges of wound care and closure.

Methods: Four patients with a total of 12 complex wounds were treated over a three year period. All of the patients were males between the ages of 28 and 61 years. The lesions were located on the buttocks $(n=5)$, chest $(n=1)$, perianal $(n=2)$, perineal $(n=2)$, and axillary regions $(n=2)$. A protocol of wide local excision, followed by negative pressure wound therapy with instillation and dwell time (NPWTi-d) to decrease bioburden and promote angiogenesis of the exposed base, and subsequent skin grafting was used. Patients remained hospitalized between procedures.

Results: The original wound area ranged from $210-540 \mathrm{~cm}^{2}$. Skin grafts of comparable sizes were taken from donor sites. The average duration of NPWTi-d placement was 3.5 days and the average time from excision to wound coverage was 4.3 days. The percent of graft uptake ranged from $70 \%-90 \%$. All patients were resolved of their local disease with no complications.

Conclusions: Surgical management of HS can be complicated by difficult closures. This case series demonstrates that wide local excision followed by NPWTi-d and skin grafting is able to achieve local resolution of disease in HS patients who have failed multiple minimally invasive therapies.

Received 08/23/2018

Review began $08 / 25 / 2018$ Review ended 09/12/2018 Published 09/17/2018

C) Copyright 2018

$\mathrm{Ge}$ et al. This is an open access article distributed under the terms of the Creative Commons Attribution License CC-BY 3.0., which permits unrestricted use, distribution, and reproduction in any medium, provided the original author and source are credited.
Categories: Dermatology, Plastic Surgery, General Surgery

Keywords: wound healing, hidradenitis suppurativa, negative pressure wound therapy with instillation and dwell, npwti-d, skin grafting

\section{Introduction}

Hidradenitis suppurativa (HS) is a chronic inflammatory disease that selectively impacts apocrine gland-bearing skin and most frequently involves the skin of intertriginous areas, such as the axillary, groin, anogenital, and inframammary regions. HS is characterized by recurrent 
and painful flares that significantly impair the quality of life and negatively impact the social, psychological, and sexual health of affected individuals [1-2]. Presentations of HS range from mild, such as subcutaneous nodules with moderate inflammation that regress without draining, to severe, where patients experience painful, diffuse abscesses that eventually coalesce to form sinus tracts containing foul-smelling, purulent, or serosanguinous drainage. Severe presentations can eventually lead to subsequent scarring and disfigurement. Early stage HS may resemble other disorders, often delaying diagnosis and treatment [3]. The true prevalence of HS is unknown, but estimates indicate that as high as $4 \%$ of the population may be affected, with the highest incidence in the second and third decades of life. Women are three times more likely to develop HS than men, and African Americans are more likely to be affected than other ethnicities [2].

Clinically, the Hurley and Sartorius systems have been used to stage HS lesions. The Hurley staging system is divided into three stages based on the presence and extent of disease. Stage I is comprised of single episodes of abscess growth without sinus tract formation, whereas stage II is characterized by recurrent episodes of abscess formation with sinus tract development and scarring. Finally, stage III encompasses diffuse abscess formation with the development of interconnected sinus tracts across entire regions of tissue and widespread scarring [4]. The Sartorius scoring system is more complex and incorporates the anatomical distribution, number, and type of lesions, as well as the distance and presence of normal tissue between lesions [5].

Negative pressure wound therapy (NPWT), most commonly used in the management of open wounds, has been adapted to treat Hurley stage II and III HS in conjunction with surgical debridement. NPWT employs a vacuum controlled system to create a subatmospheric layer of pressure that is distributed symmetrically over a wound through an open cell foam dressing. NPWT is simple yet effective for the promotion of granulation tissue formation in acute and chronic wounds, and has been shown to reduce wound contamination and promote angiogenesis [6]. NPWT with instillation and dwell time (NPWTi-d) is a new form of NPWT initially used for the treatment of infected orthopedic prosthesis [7-8]. This system intermittently pauses NPWT to instill an antiseptic solution on the wound and allows the solution to stay on the wound for a predetermined period of time. NPWT is resumed at the end of this dwell phase.

In this study, we report a series of patients with severe HS complicated by wound contamination treated with combined wide surgical resection and NPWTi-d. Our aim is to introduce a new treatment option in the management of this challenging disease.

\section{Materials And Methods}

Participants included four male African American patients, aged 28-61 years, body mass index (BMI) 19.3-36.7, with a total of 12 complex and contaminated wounds due to longstanding Hurley stage III HS. Five on the buttocks, one on the chest, and two in each of the perianal, perineal, and axillary regions. All patients developed significant sinus tracts, abscesses, and fistulization. All patients had failed multiple courses of topical and systemic therapies, requiring definitive treatment with wide surgical excision and debridement.

All procedures were completed between 2014 and 2017. During the first operation, excision of all diseased skin, abscesses, and fistula tracts was carried down to the level of subcutaneous tissue. Tissue advancement rearrangement was done to minimize the size of the wounds. Vacuum-assisted closure (VAC) VeraFlo ${ }^{\mathrm{TM}}$ wound dressing system (NPWTi-d; VAC Instill ${ }^{\circledR}$ Wound Therapy, KCI, an Acelity company, San Antonio, TX) was used to cover the wounds between the initial debridement and definitive closure steps. The instillation agent used was Clorpactin WCS-90 chlorine powder (United-Guardian, Inc., Hauppauge, NY) in one-liter sterile 
water. The suction was set to $-125 \mathrm{mmHg}$, cycle frequency was set to 3.5 hours, and irrigation dwell time was set to 10 minutes. All patients were admitted to the plastic surgery inpatient service between procedures and placed on bed rest. Patients were brought back to the operating room on postoperative day three to six for split-thickness skin grafting. Upon NPWTi-d takedown during the second operation, all wounds appeared to be in good condition and clean, with robust granulating base and no purulent drainage (Figure 1). Tissue laxity around the wounds allowed additional tissue advancement to further minimize the size of the exposed base and donor site morbidity in some cases. After advancement, tissues were secured with 3-0 absorbable sutures $\left(V{ }^{2}{ }^{\circledR}\right.$, Ethicon, Sommerville, NJ). Skin grafts were harvested at 1/12,000 inch from the thighs, meshed to 1.5:1, and secured over the wounds with 3-0 chromic sutures (Ethicon, Sommerville, NJ) (Figure 2). Silver foam dressing and NPWT without irrigation (VAC $\mathrm{ULTA}^{\mathrm{TM}}$, San Antonio, TX) were placed over the grafts at $-125 \mathrm{mmHg}$ continuous suction. Donor sites were covered with Aquacel ${ }^{\circledR}$ (Convatec, Oklahoma City, OK), Tegaderm ${ }^{\mathrm{TM}}$ (3M, St. Paul, $\mathrm{MN})$, and elastic bandage.
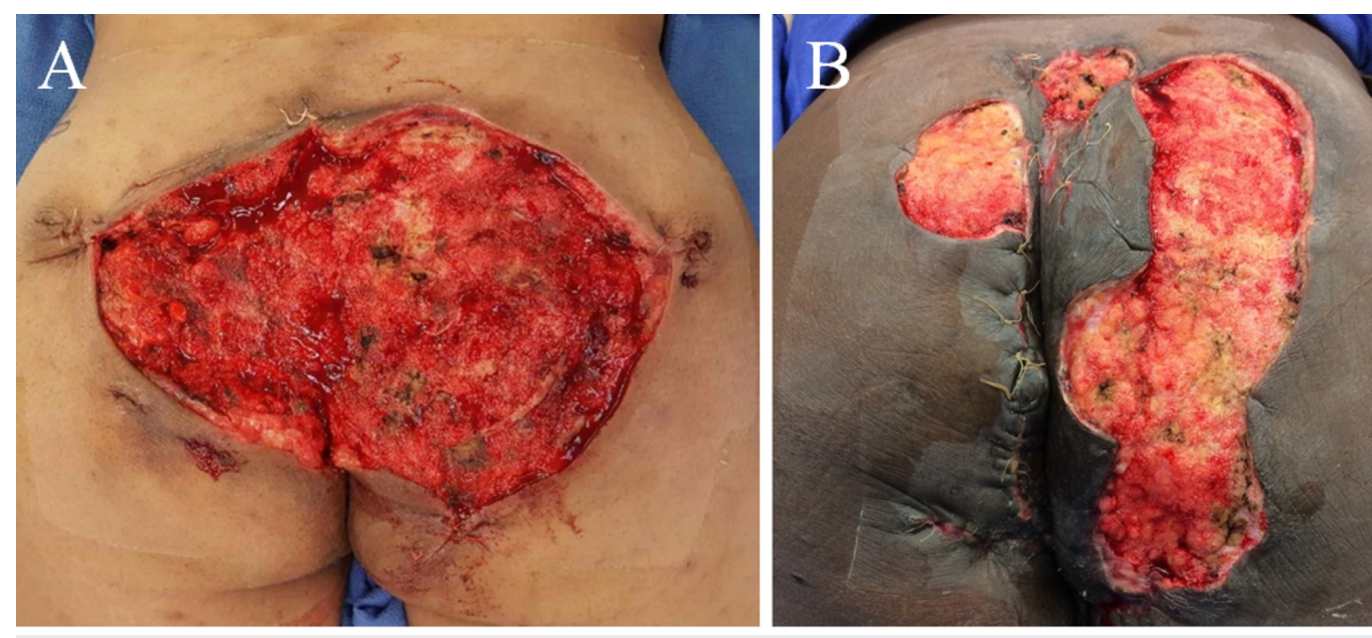

FIGURE 1: Upon negative pressure wound therapy with instillation and dwell time takedown during the second operation, patients demonstrated healthy pink granulating base without significant fibrinous tissue, slough, or eschar.

Patient A was treated for diffuse bilateral buttocks and perianal involvement. Patient B suffered from extensive hidradenitis suppurativa lesions in the left perianal, perineal, and bilateral buttocks regions.
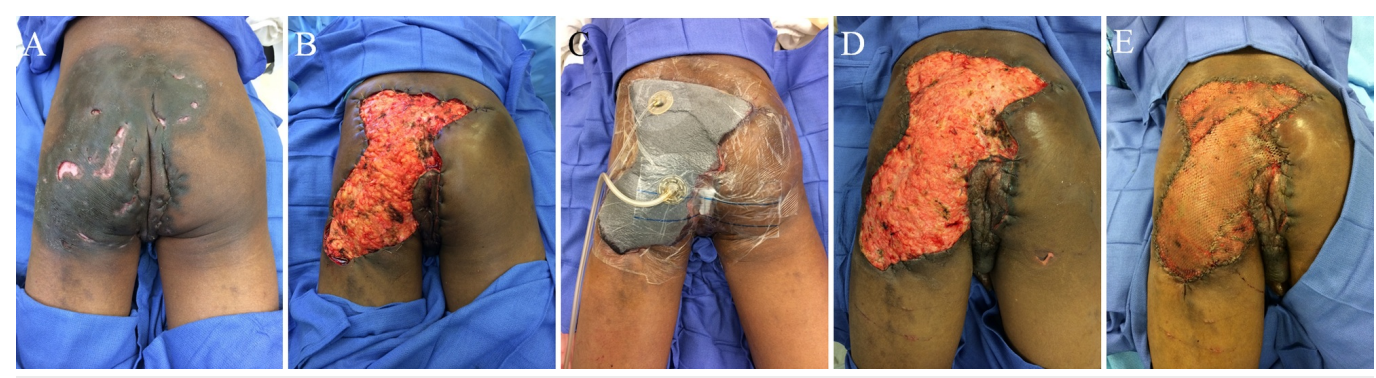

FIGURE 2: Patient with extensive disease in the left buttock and perineum (A) who underwent wide local excision with tissue advancement $(B)$ and negative pressure wound therapy 


\section{Cureus}

with instillation and dwell time (NPTWi-d) placement (C); healthy granulating base was found upon NPTWi-d takedown

(D) and autologous skin was grafted onto the wound base (E)

\section{Results}

NPWTi-d did not need to be changed between surgeries and the total number of surgeries for each patient was two. First for excision and NPWTi-d placement and second for the NPWTi-d takedown and skin grafting (Table 1). The volume of irrigant varied between 22-60 ml depending on the size of the wounds. The duration of NPTWi-d placement varied between three to four days with an average of 3.5 days. The interval from excision to complete wound coverage with grafting varied between three to six days with an average of 4.3 days. One patient received skin grafting six days after the initial surgery due to scheduling difficulties; however, his NPWTi-d was removed on postoperative day three and the wound was managed with wet to dry dressing changes until the second procedure. The original wound size measured between 210-540 cm2, skin grafts of similar sizes were harvested from donor sites, and the percent of graft uptake ranged from 70\%-90\%. The average time from skin grafting to complete healing ranged from eight to 17 weeks. NPWTi-d failure, wound infections, and surgical complications were not observed in any of the patients. None of the patients required repeat hospitalization, reoperation, or regrafting. Patients were followed up to four years postoperatively and all were doing well with no recurrence of local disease (Figure 3). 


\section{Cureus}

\begin{tabular}{|c|c|c|c|c|}
\hline Patients & I & II & III & IV \\
\hline Age & 34 & 28 & 61 & 51 \\
\hline Sex & $\mathrm{M}$ & $\mathrm{M}$ & $\mathrm{M}$ & $\mathrm{M}$ \\
\hline Body mass index $\left(\mathrm{kg} / \mathrm{m}^{2}\right)$ & 24.3 & 33.1 & 19.3 & 36.7 \\
\hline Location of disease & $\begin{array}{l}\text { Bilateral buttocks, } \\
\text { perianal }\end{array}$ & $\begin{array}{l}\text { Bilateral axilla, } \\
\text { left chest }\end{array}$ & $\begin{array}{l}\text { Left buttock, } \\
\text { perineum }\end{array}$ & $\begin{array}{l}\text { Left perianal, perineal, } \\
\text { bilateral buttocks }\end{array}$ \\
\hline Area of disease $\left(\mathrm{cm}^{2}\right)$ & 391 & 246 & 540 & 210 \\
\hline $\begin{array}{l}\text { Vacuum Assisted Closure settings } \\
\text { (mmHg, hr) }\end{array}$ & $-125,3.5$ & $-125,3.5$ & $-125,3.5$ & $-125,3.5$ \\
\hline Dwell time (min) & 10 & 10 & 10 & 10 \\
\hline Instillation volume (mL) & 36 & 22 & 22 & 60 \\
\hline $\begin{array}{l}\text { Duration of Vacuum Assisted } \\
\text { Closure placement (d) }\end{array}$ & 4 & 3 & 4 & 3 \\
\hline Duration between surgeries (d) & 4 & 6 & 4 & 3 \\
\hline Time to complete healing (w) & 14 & 13 & 8 & 17 \\
\hline Percent graft uptake & $80 \%$ & $90 \%$ & $90 \%$ & $70 \%$ \\
\hline
\end{tabular}

\section{TABLE 1: Summary of patient outcomes}
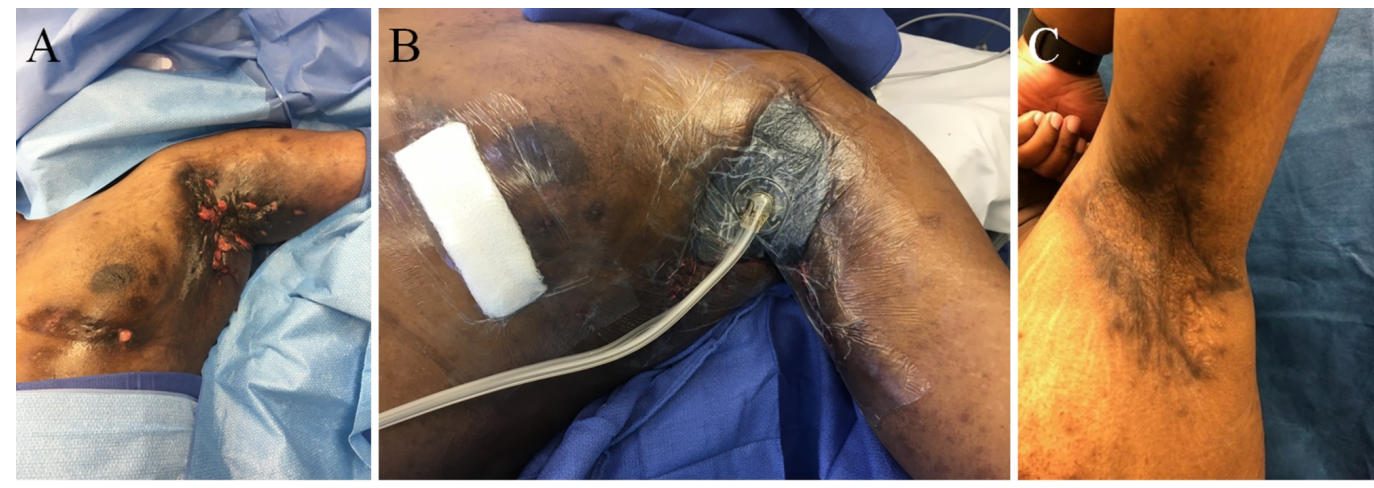

FIGURE 3: Patient with severe bilateral axillary disease with left chest involvement (A) who underwent local excision, tissue advancement, and negative pressure wound therapy with instillation and dwell time (NPTWi-d) placement (B); postoperative 21 months with no local disease recurrence following successful autologous skin grafting (C) 


\section{Discussion}

HS is a debilitating disease with physical and psychosocial limitations, made worse by severe wound contamination that slows healing and limits therapeutic options. To date, severe HS continues to be a debilitating condition that remains difficult to treat. Biofilms can be observed in the sinus tracts of $67 \%$ of chronic HS lesions and contributes to the difficult nature of the disease [9]. Topical therapies such as clindamycin ointment have been used in patients with superficial HS, but such treatment displays poor efficacy for deeper lesions and abscesses [10]. Systemic antibiotics such as clindamycin/rifampin, metronidazole, and ertapenem have also been used to treat HS and demonstrated variable response and relapse rates [11-12]. Studies utilizing oral retinoids, methotrexate, and dapsone have determined them ineffective as firstline therapies and should only be used in combination with other therapeutic agents [13-15]. Intralesional steroid injections have successfully reduced inflammation in acute HS flares and systemic steroids have shown satisfying results in severe or refractory cases, but are not recommended for long-term use [16-17]. Biologic agents, such as the TNF $\alpha$ antagonists infliximab and adalimumab, have shown promising results in reducing the severity and frequency of recurrences in patients with severe or refractory HS, but are associated with serious adverse events [18-20]. Thus, a multidisciplinary therapeutic approach with combined medical and surgical management has remained necessary for many patients with severe or diffuse HS, but introduces immense wound closure and healing challenges.

NPTWi-d therapy has been demonstrated as one of the most effective methods in assisting wound closure. In addition to improving wound granulation and vascularization, the use of NPWTi-d in the management of complex wounds significantly reduces wound contamination by continuous removal of bacteria and disruption of bacterial attachment to tissues. This results in a reduced bacterial load in wounds complicated by virulent pathogens, and even wounds that harbor pathogens with attachment through biofilms or multidrug resistance [2122]. In in vivo and in vitro wound models, NPWTi-d has proven superior to NPWT alone through reduction of bioburden in artificially infected wounds [23-24]. In human studies, NPWTi-d has shown to be effective in decontaminating chronically infected wounds in the lower extremities, leading to successful graft uptake even in patients with significant comorbidities [25-26]. While the increased technological complexity of the NPWTi-d system may appear off-putting due to the need for hospitalization for intermittent wound irrigation and increased hospital staffing requirements, NPWTi-d has proven overall advantageous by reducing the number of procedures required for closure in abdominal wounds, hospitalization time, episodes of hospitalization, and total expense of treatment [27].

Instillation solution selection is critical to accomplish cleansing and granulation goals of NPWTi-d. Several solutions can be used with the NPWTi-d system, such as Dakins solution, silver nitrate, normal saline, polyhexanide, and protosan. Successful wound closure has been reported with each of these solutions but the presence of active infection or risk of infection should be taken into consideration when choosing among solutions. Clean wounds can be managed only with saline but contaminated or infected wounds may require an instillation with local antiseptic properties [28]. Clorapactin was chosen due to its powerful germicide, fungicide, and virucide properties. Yet it is nontoxic and nonirritating when used in therapeutic dosages. It is an excellent oxidizing agent and exhibits pronounced wetting, penetrating, and detergent properties. Additionally, it is widely available at a low cost [29].

Other surgical treatment methods for HS include the use of local or perforator flaps and recycled skin grafts [30]. Local and perforator flaps yield successful results in select patient populations, such as patients with small to moderate sized defects. However, both of these methods have significant morbidities and donor site limitations. The excision of advanced HS creates large skin defects, local or perforator flaps may not be adequate for the reconstruction of these defects. The recycled skin graft method was described to decrease the donor site 
morbidity associated with split thickness skin grafts [30]. The skin over the diseased area is harvested as a split thickness skin graft and reused to cover the defect resulting from the surgical excision of HS. This is a brilliant method that significantly decreases the donor site morbidity if immediate reconstruction is planned. However, NPWTi-d was initially applied to the defects to stimulate granulation formation and reduced bioburden prior to skin grafting in this study. Wounds were covered with skin grafts in a second stage operation. Moreover, reconstructing the defect with the same, chronically infected skin may theoretically increase the risk of postoperative wound infection leading to graft loss.

NPWTi-d is a recent technique successful in other patient populations, most notably orthopedic patients with prosthesis infections [21-22], and may provide an additional option for the management of Hurley stage III HS. This case series offers support for the use of NPWTi-d for severe HS requiring surgical excision and subsequent skin grafting. All patients achieved adequate wound decontamination and tissue granulation within days of excision, shortening the length of their hospitalization. Furthermore, all patients demonstrated successful graft uptake and healed with resolution of local disease. The limitations of our study include: a small sample size; end points based on clinical parameters alone, such as wound appearance, graft uptake, and patient outcome; and that bioburden before and after NPWTi-d was not measured. Additional studies with a larger sample size and microbiological in addition to clinical parameters may be needed to better characterize the use of NPWTi-d in patients with severe and diffuse HS.

\section{Conclusions}

NPWTi-d is a highly successful tool for preparing wounds for closure. It protects the newly developing granulation tissue base and decreases the risk of wound infections. Our study demonstrates that the use of NPWTi-d for the treatment of Hurley stage III HS is not only safe and simple, but also produces excellent outcomes.

\section{Additional Information}

\section{Disclosures}

Human subjects: Consent was obtained by all participants in this study. Animal subjects: All authors have confirmed that this study did not involve animal subjects or tissue. Conflicts of interest: In compliance with the ICMJE uniform disclosure form, all authors declare the following: Payment/services info: All authors have declared that no financial support was received from any organization for the submitted work. Financial relationships: Ronald P. Silverman, M.D. declare(s) employment from Acelity L.P., Inc., . Ronald P Silverman, M.D. serves as the Chief Medical Officer and Senior Vice President of Acelity L.P., Inc., that is producing the negative pressure wound dressing system used in the research being reported. . Other relationships: All authors have declared that there are no other relationships or activities that could appear to have influenced the submitted work.

\section{References}

1. Kouris A, Platsidaki E, Christodoulou C, et al.: Quality of life and psychosocial implications in patients with hidradenitis suppurativa. Dermatology. 2016, 232:687-91. 10.1159/000453355

2. Alikhan A, Lynch PJ, Eisen DB: Hidradenitis suppurativa: a comprehensive review . J Am Acad Dermatol. 2009, 60:539-61. 10.1016/j.jaad.2008.11.911

3. Margesson LJ, Danby FW: Hidradenitis suppurativa. Best Pract Res Clin Obstet Gynaecol. 2014, 28:1013-27. 10.1016/j.bpobgyn.2014.07.012

4. Hurley H: Dermatological Surgery: Principles and Practice. Roenigk RK, Roenigk HH (ed): Marcel Dekker Inc, New York; 1989.

5. Sartorius K, Lapins J, Emtestam L, Jemec GB: Suggestions for uniform outcome variables when 
reporting treatment effects in hidradenitis suppurativa. Br J Dermatol. 2003, 149:211-3. 10.1046/j.1365-2133.2003.05390.x

6. Argenta LC, Morykwas MJ: Vacuum-assisted closure: a new method for wound control and treatment: clinical experience. Ann Plast Surg. 1997, 38:563-76.

7. Kurtz SM, Lau E, Watson H, Schmier JK, Parvizi J: Economic burden of periprosthetic joint infection in the United States. J Arthroplasty. 2012, 27:61-5. 10.1016/j.arth.2012.02.022

8. Collins I, Wilson-MacDonald J, Chami G, et al.: The diagnosis and management of infection following instrumented spinal fusion. Eur Spine J. 2008, 17:445-50. 10.1007/s00586-007-05598

9. Ring HC, Bay L, Nilsson M, et al.: Bacterial biofilm in chronic lesions of hidradenitis suppurativa. Br J Dermatol. 2017, 176:993-1000. 10.1111/bjd.15007

10. Clemmensen OJ: Topical treatment of hidradenitis suppurativa with clindamycin . Int J Dermatol. 1983, 22:325-8. 10.1111/j.1365-4362.1983.tb02150.x

11. Gener G, Canoui-Poitrine F, Revuz JE, et al.: Combination therapy with clindamycin and rifampicin for hidradenitis suppurativa: a series of 116 consecutive patients. Dermatology. 2009, 219:148-54. 10.1159/000228334

12. Join-Lambert O, Coignard-Biehler H, Jais JP, et al.: Efficacy of ertapenem in severe hidradenitis suppurativa: a pilot study in a cohort of 30 consecutive patients. J Antimicrob Chemother. 2016, 71:513-20. 10.1093/jac/dkv361

13. Tan MG, Shear NH, Walsh S, Alhusayen R: Acitretin: monotherapy or combined therapy for hidradenitis suppurativa?. J Cutan Med Surg. 2016, 21:48-53. 10.1177/1203475416659858

14. Yazdanyar S, Boer J, Ingvarsson G, Szepietowski JC, Jemec GB: Dapsone therapy for hidradenitis suppurativa: a series of 24 patients. Dermatology. 2011, 222:342-6. $10.1159 / 000329023$

15. Jemec GB: Methotrexate is of limited value in the treatment of hidradenitis suppurativa . Clin Exp Dermatol. 2002, 27:528-9. 10.1046/j.1365-2230.2002.11125.x

16. Hidradenitis Suppurativa. Jemec GB, Revuz J, Leyden JJ (ed): Springer, Berlin, Germany; 2006.

17. Zouboulis CC, Desai N, Emtestam L, et al.: European S1 guideline for the treatment of hidradenitis suppurativa/acne inversa. J Eur Acad Dermatol Venereol. 2015, 29:619-44. 10.1111/jdv.12966

18. Delage M, Samimi M, Atlan M, Machet L, Lorette G, Maruani A: Efficacy of infliximab for hidradenitis suppurativa: assessment of clinical and biological inflammatory markers. Acta Derm Venereol. 2011, 91:169-71. 10.2340/00015555-1025

19. Arenbergerova M, Gkalpakiotis S, Arenberger P: Effective long-term control of refractory hidradenitis suppurativa with adalimumab after failure of conventional therapy. Int J Dermatol. 2010, 49:1445-9. 10.1111/j.1365-4632.2010.04638.x

20. Sotiriou E, Apalla Z, Vakirlis E, Ioannides D: Efficacy of adalimumab in recalcitrant hidradenitis suppurativa. Eur J Dermatol. 2009, 1:180-1. 10.1684/ejd.2008.0599

21. Yang C, Goss SG, Alcantara S, Schultz G, Lantis Ii JC: Effect of negative pressure wound therapy with instillation on bioburden in chronically infected wounds. Wounds. 2017, 29:2406.

22. Raad W, Lantis JC 2nd, Tyrie L, Gendics C, Todd G: Vacuum-assisted closure instill as a method of sterilizing massive venous stasis wounds prior to split thickness skin graft placement. Int Wound J. 2010, 7:81-5. 10.1111/j.1742-481X.2010.00658.x

23. Davis K, Bills J, Barker J, Kim P, Lavery L: Simultaneous irrigation and negative pressure wound therapy enhances wound healing and reduces wound bioburden in a porcine model. Wound Repair Regen. 2013, 21:869-75. 10.1111/wrr.12104

24. Matiasek J, Domig KJ, Djedovic G, Babeluk R, Assadian O: The effect of negative pressure wound therapy with antibacterial dressings or antiseptics on an in vitro wound model. J Wound Care. 2017, 26:236-42. 10.12968/jowc.2017.26.5.236

25. Goss SG, Schwartz JA, Facchin F, Avdagic E, Gendics C, Lantis JC 2nd: Negative pressure wound therapy with instillation (NPWTi-d) better reduces post-debridement bioburden in chronically infected lower extremity wounds than NPWT alone. J Am Coll Clin Wound Spec. 2014, 4:74-80. 10.1016/j.jccw.2014.02.001

26. Matiasek J, Djedovic G, Unger L, et al.: Outcomes for split-thickness skin transplantation in high-risk patients using octenidine. J Wound Care. 2015, 24:10-2.

10.12968/jowc.2015.24.Sup6.S8

27. Deleyto E, García-Ruano A, González-López JR: Negative pressure wound therapy with 


\section{Cureus}

instillation, a cost-effective treatment for abdominal mesh exposure. Hernia. 2018, 22:311-8. 10.1007/s10029-017-1691-y

28. Gupta S, Gabriel A, Lantis J, Téot L: Clinical recommendations and practical guide for negative pressure wound therapy with instillation. Int Wound J. 2016, 13:159-74.

10.1111/iwj.12452

29. Zwerling M: Clorpactin WCS-90: a new antiseptic; preliminary report. AMA Arch Otolaryngol. 1955, 62:157-8. 10.1001/archotol.1955.03830020039008

30. Kuo HW, Ohara K: Surgical treatment of chronic gluteal hidradenitis suppurativa: reused skin graft technique. Dermatol Surg. 2003, 29:173-8. 10.1046/j.1524-4725.2003.29044.x 